Document downloaded from:

http://hdl.handle.net/10251/54638

This paper must be cited as:

Gozálvez Serrano, D.; Gimenez Gandia, JJ.; Gómez Barquero, D.; Cardona Marcet, N. (2013). Rotated constellations for improved time and frequency diversity in DVB-NGH. IEEE Transactions on Broadcasting. 59(2):298-305. doi:10.1109/TBC.2013.2252862.

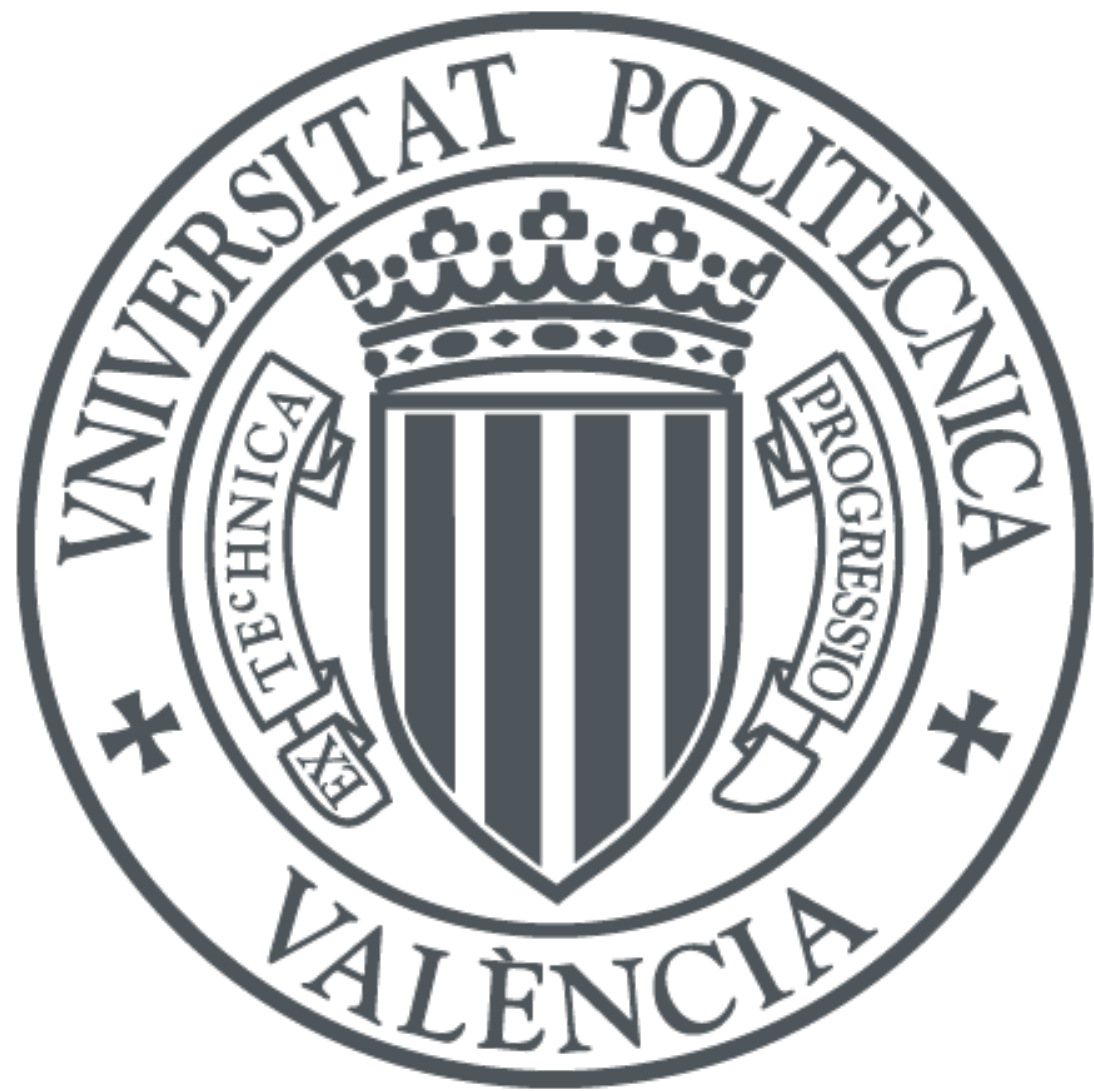

The final publication is available at

http://dx.doi.org/10.1109/TBC.2013.2252862

Copyright Institute of Electrical and Electronics Engineers (IEEE)

Additional Information

"(C) 2013 IEEE. Personal use of this material is permitted. Permission from IEEE must be obtained for all other uses, in any current or future media, including reprinting/republishing this material for advertising or promotional purposes, creating new collective works, for resale or redistribution to servers or lists, or reuse of any copyrighted component of this work in other works." 


\title{
Rotated Constellations for Improved Time and Frequency Diversity in DVB-NGH
}

\author{
David Gozálvez, Jordi Joan Giménez, David Gómez-Barquero, and Narcís Cardona \\ Instituto de Telecomunicaciones y Aplicaciones Multimedia (iTEAM) \\ Universitat Politècnica de València (UPV) \\ Email: \{dagoser, jorgigan, dagobar, ncardona\}@iteam.upv.es
}

\begin{abstract}
In this paper, we investigate the potential gains that can be obtained with rotated constellations in DVB-NGH, the next generation mobile broadcasting standard. Rotated constellations exploit the concept of signal-space diversity (SSD) to increase the diversity order of bit-interleaved coded modulation (BICM) at the expense of higher demodulation complexity without the need of additional transmission power or bandwidth. Two-dimensional rotated constellations (2DRC) were originally included in DVBT2 (Terrestrial 2nd generation) to improve the reception robustness in fading channels. DVB-NGH inherits the same 2DRC from DVB-T2 and includes four-dimensional rotated constellations (4DRC) for certain configurations. Moreover, the standard has adopted a new component interleaver optimized for the utilization of rotated constellations with long time interleaving (TI) and time-frequency slicing (TFS). In this context, the additional robustness of rotated constellations is very interesting to counter the presence of signal outages in the time and frequency domains. To investigate the potential gains of $2 \mathrm{DRC}$ and $4 \mathrm{DRC}$ we employ an information-theoretic approach based on mutual information as well as physical layer simulations in DVB-NGH systems. The results reveal that rotated constellations are important to increase the diversity gains of long TI and TFS, and also to reduce the zapping time perceived by the users.
\end{abstract}

Index Terms-Bit-interleaved coded modulation, DVB-NGH, DVB-T2, time interleaving, rotated constellations, time-frequency slicing.

\section{INTRODUCTION}

The incorporation of diversity techniques is key to improve the reception robustness and to extend the coverage area in the next generation of mobile broadcasting systems [1]. Binary-interleaved coded modulation (BICM) with orthogonal frequency division multiplexing (OFDM) is currently the preferred option for terrestrial mobile broadcasting. These systems rely on the combination of forward error correction (FEC) and interleaving to achieve diversity gains in the time and frequency domains [2]. Furthermore, signal-space diversity (SSD), also known as rotated constellations, can be used to increase the diversity order of BICM systems at the expense of increased demodulation complexity without the need of additional transmission power or bandwidth [3]. The main idea behind SSD is to apply a certain rotation to a classical signal constellation, so that the binary information is transmitted simultaneously in different components, with each component experiencing independent fading. In order to ensure that each component undergoes independent fading, it is necessary to employ an interleaver that separates the components in the time and frequency domains after the rotation. Increasing the dimension of the rotation improves the diversity order but requires a higher demodulation complexity due to the fact that all the components must be demodulated together in order to retrieve the binary information [4].

The second generation European standard for terrestrial broadcasting, known as DVB-T2, incorporates low density parity check (LDPC) codes as well as two-dimensional rotated constellations (2DRC) [5]. The adoption of LDPC codes enables a performance within $1 \mathrm{~dB}$ of the Shannon limit in AWGN [6], whereas the use of 2DRC improves the reception robustness in fading channels. In particular, the reception of strong line-of-sight (LoS) echoes coming from different transmitters in single frequency networks (SFN) might result in the presence of deep notches in the frequency response of the channel. These notches can erase a significant percentage of subcarriers and prevent the decoding of services even when the average carrier-to-noise ratio (CNR) is well above the reception threshold [7]. The gain of 2DRC in DVB-T2 is maximum with lower order constellations (e.g. QPSK) and higher code rates (e.g. $>1 / 2$ ), and when the percentage of erasures in the channel increases [8].

DVB-NGH, the next generation European standard for terrestrial mobile broadcasting [9], inherits the LDPC codes and 2DRC from DVB-T2, and also incorporates four-dimensional rotated constellations (4DRC) for certain configurations. Compared to DVB-T2, the major improvement in reception robustness is achieved by the inclusion of time, frequency and polarization diversity by means of long time interleaving (TI), time-frequency slicing (TFS) and cross-polar multiple-input multiple-output (MIMO) [10]. Long TI and TFS are used to compensate for the large signal variations encountered along the user trajectory, and between different RF channels, respectively. In the time domain, the signal outages caused by shadowing might result in the loss of entire information frames [11], whereas in the frequency domain, the presence of interference from neighbouring networks added to the frequency dependency of the transmitter site, the receiver and the propagation channel, might cause the loss of entire RF channels [12]. DVB-NGH has also adopted a component interleaver optimized for the combination of rotated constellations with long TI and TFS.

The work presented in this paper investigates the potential gains of the 2DRC and the 4DRC adopted in DVB-NGH from a information-theoretic point of view based on mutual information, and also by means of physical layer simulations. 
Although the theoretical framework has been established in the past [13], previous investigations do not consider the presence of outages in the received signal and generally assume a Gaussian or Rayleigh channel. In comparison, the main novelty of the paper is the investigation of the capabilities of rotated constellations to cope with signal outages in the time and frequency domains, and the performance gains that can be achieved by combining rotated constellations with long TI and TFS in DVB-NGH systems. Such an investigation is not available in the literature and was not performed during the standardization process of DVB-NGH.

The paper is structured as follows. In Section II, we give a brief introduction to DVB-NGH and describe the rotated constellations and the component interleaver that have been adopted in the standard. In Section III, we show the potential gains of rotated constellations based on mutual information. In Section IV, we present simulation results that illustrate the improvement achieved by rotated constellations in DVBNGH systems. Finally, we give some concluding remarks in Section V.

\section{Rotated CONSTELlations IN DVB-NGH}

\section{A. BICM Module}

The BICM module in the DVB-NGH standard is based on a sub-set of components from the DVB-T2 standard [14] intended to lower the receiver complexity and power consumption, with additional components for extended coverage and increased robustness in mobile reception. At the FEC level, DVB-NGH has adopted a set of LDPC codes with rates uniformly spaced between $1 / 5$ and $11 / 15$. At the constellation level, it has inherited the same 2DRC of DVB-T2 for configurations using QPSK, 16QAM, and 64QAM constellations, and has also included 4DRC for configurations using QPSK and code rates higher than $1 / 2$.

DVB-NGH adopts a combination of block and convolutional interleaving for the provision of TI at the physical layer. The block interleaver performs intra-frame interleaving in the order of hundreds of milliseconds in order to accomplish a uniform interleaving of information symbols within the frames. The convolutional interleaver performs inter-frame interleaving up to several seconds by distributing the information symbols of each FEC codeword across multiple frames. When TFS is used, the frames are multiplexed over several RF channels by means of frequency hopping and time slicing. In particular, the frames are transmitted sequentially in the RF channels that constitute one TFS multiplex. This allows extending the interleaving beyond one RF channel and across hundreds of $\mathrm{MHz}$ in the frequency domain. Reception of TFS services can be performed with a single tuner, but requires a time interval between the slices of the same service in order to perform frequency hopping. In the case of HDTV, the time interval needed for frequency hopping cannot be guaranteed due to the high bit rate of this kind of services, and a second tuner is required for TFS operation. However, this is not a problem in DVB-NGH, as the lower bit rates of mobile TV services allow receivers to perform frame hopping with a single tuner [10].

In the terrestrial profiles of DVB-NGH, the convolutional interleaver accomplishes a uniform interleaving of information

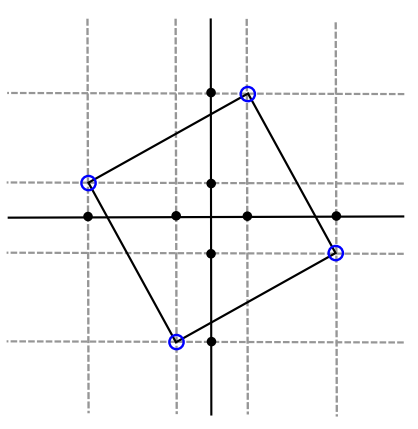

QPSK 2DRC

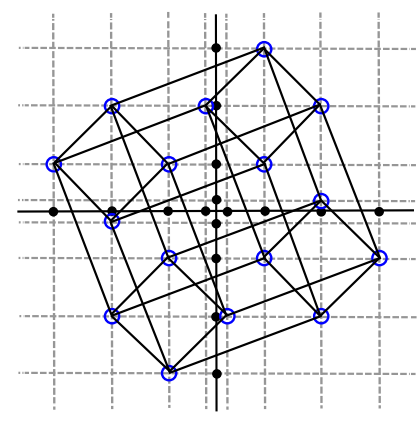

QPSK 4DRC
Fig. 1. Two-dimensional and four-dimensional rotated constellations adopted in DVB-NGH.

across frames, whereas in the hybrid terrestrial-satellite profiles, it can also perform uniform-late interleaving in order to reduce the zapping time at the expense of reception robustness (time diversity). In this case, a larger portion of each codeword is sent in a late frame with a duration of typically hundreds of milliseconds, so that the receiver can start decoding the service immediately after the reception of the late frame. This is usually known as fast zapping. With fast zapping, the missing parts of the codewords that are not transmitted in the late frame are seen as erasures at the input of the FEC decoder. Therefore, the capability to perform fast zapping in receivers is determined by the robustness of the FEC code against the presence of erasures. At the same time, the utilization of nonuniform interleaving might reduce the reception robustness in mobile channels as a result of worse time diversity. The tradeoff between zapping time and time diversity is determined by the percentage of information that is carried in the late part. It should be noted that uniform-late configurations with more than $50 \%$ of information in the late part are generally not practical due the high performance degradation that occurs in mobile channels.

\section{B. Constellation rotation}

In BICM systems like DVB-NGH, a sequence of information bits is encoded using a FEC code and is then passed through a bitwise interleaver. After this, each block of $m$ coded interleaved bits $c_{l}, l=1, \ldots, m$ is mapped to a complex data symbol $u$ in a conventional QAM constellation $\mathcal{A}$ [2]. In order to achieve SSD, a rotation is applied to the constellation symbols so that each block of $m$ bits is spread across the $D$ components of the rotated symbol $\mathbf{x}=\left[x_{1}, x_{2}, \ldots, x_{D}\right]$ in the $D$-dimensional rotated constellation $\Psi$ [3]. Then, a component interleaving is used to separate the components of the rotated symbol in the time and frequency domains so that each component faces an independent fading realization. The 2DRC and 4DRC adopted in DVB-NGH can be seen in Fig. 1.

When using conventional QAM constellations, each signal component in the imaginary plane, in-phase (I) or quadrature (Q), carries half of the binary information. Therefore, it is not possible to retrieve the whole binary content when one of 
Erasure event spanning four frames with 2DRC

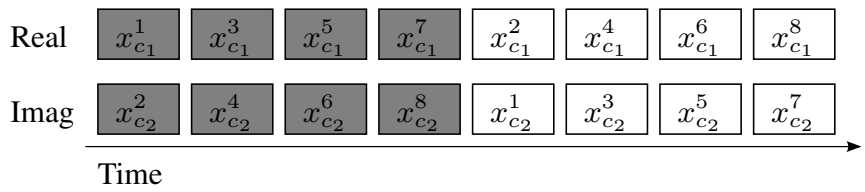

Erasure event spanning six frames with 4DRC

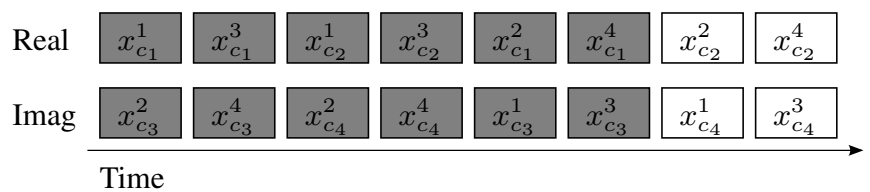

Fig. 2. Interleaving of components with inter-frame interleaving across eight frames.

the two components is erased. In the case of $2 \mathrm{DRC}$, both I and Q components carry all the binary information, and thus, it is possible to retrieve the information even if one of the two components is deeply faded or erased. In DVB-T2 and DVB-NGH, the rotation of 2DRC has been optimized for each constellation as a good compromise between the pairwise error probability over fading channels in high signalto-noise regions, and the bit error probability when one of the components I or Q is erased [8]. With 4DRC, the rotation is applied in order to spread the binary information across four different components. In this case, the rotation has been selected to minimize the bit error probability according to physical layer simulations in Rayleigh channels.

\section{Component Interleaving}

In DVB-T2, the component $\mathrm{Q}$ of the rotated symbols is first cyclically delayed by one cell inside the FEC codewords. Then, a cascade of cell, time, and frequency interleavers separates the components in the time and frequency domains in order to reduce the fading correlation. In DVB-NGH, the cyclic Q delay has been replaced by a more sophisticated component interleaver optimized for inter-frame interleaving and TFS. The component interleaver is designed in such a manner that, with inter-frame interleaving, the different components of each rotated symbol end up being transmitted in different frames, with the maximum possible separation between components inside the interleaving duration. If TFS is used, the component interleaver assigns each component to a different RF channel in a cyclical manner.

The main advantage of rotated constellations when they are used in combination with inter-frame interleaving and TFS, is that they can reduce the loss of information against signal outages in the time and frequency domain. We will use figures 2 and 3 as an example to illustrate the improvement that can be achieved in DVB-NGH by using rotated constellations. In Fig. 2, we show the distribution of components when the TI extends across eight frames. In the figure, $x_{c_{i}}^{j}$ denotes the component $i$ of the rotated symbol $j$. We can see that, with 2DRC, a signal outage extending across four or less consecutive frames in the time domain results in only one of the two components, I or Q, being erased for any symbol. Therefore, it is possible to retrieve all the information at the
Erasure event spanning one RF channel with 2DRC

\begin{tabular}{|c|c|c|c|c|c|c|c|c|}
\hline & RF1 & RF2 & RF3 & RF4 & RF1 & RF2 & RF3 & RF4 \\
\hline Real & $x_{c_{1}}^{1}$ & $x_{c_{1}}^{2}$ & $x_{c_{1}}^{3}$ & $x_{c_{1}}^{4}$ & $x_{c_{1}}^{5}$ & $x_{c_{1}}^{6}$ & $x_{c_{1}}^{7}$ & $x_{c_{1}}^{8}$ \\
\hline Imag & $x_{c_{2}}^{7}$ & $x_{c_{2}}^{8}$ & $x_{c_{2}}^{1}$ & $x_{c_{2}}^{2}$ & $x_{c_{2}}^{3}$ & $x_{c_{2}}^{4}$ & $x_{c_{2}}^{5}$ & $x_{c_{2}}^{6}$ \\
\hline
\end{tabular}

Erasure event spanning three RF channel with 4DRC

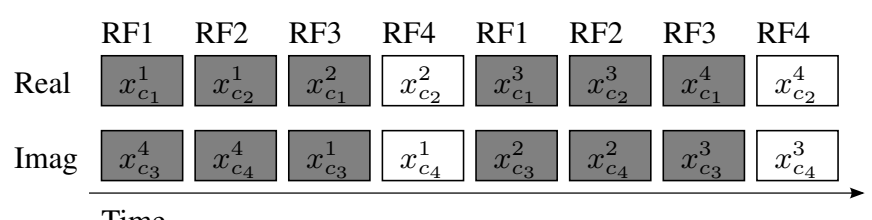

Time

Fig. 3. Interleaving of components with inter-frame interleaving and TFS across eight frames and four RF channels.

receiver side when demodulating the signal by using one of the two components that has not been erased by the channel. On the contrary, the loss of five or more consecutive frames causes the erasure of both components for some symbols. With 4DRC, it is possible to suffer an outage extending across six or less consecutive frames and still receive one component of each rotated symbol. Generally speaking, the maximum number of frames, $E_{\text {frame }}$, that can be lost while receiving at least one component of each symbol is:

$$
E_{\text {frame }}=\left\lfloor N_{\text {int }}\left(1-\frac{1}{D}\right)\right\rfloor,
$$

where $N_{\text {int }}$ denotes the number of frames covered by the interleaving duration and $D$ denotes the dimension of the rotation. The previous analysis can be extended to the case when uniform-late interleaving is used. Let us assume a uniform-late configuration with the $50 \%$ of information being transmitted in the late frame. In this case, the symbols corresponding to the first four frames in the previous example will be carried in a single frame that can be used by the receiver to start decoding the service before the reception of all the information symbols. With rotated constellations, the receiver has at least one component of each symbol after receiving the late frame, improving the probabilities to decode the service. The use of rotated constellation might also reduce the performance degradation of uniform-late interleaving in terms of time diversity by reducing the loss of information when the late frame is in deep fading.

In Fig. 3, we show the distribution of components when the frames are transmitted across four different RF channels by means of TFS. Here, we focus on the loss of one or multiple RF channels caused, for example, by the presence of interference from neighbouring networks. The distribution performed by the component and convolutional interleavers places the component of each rotated symbol in a different $\mathrm{RF}$ channel. We can see that the maximum number of RF channels, $E_{\text {chan }}$, that can be lost while receiving at least one component of each symbol is equal to the dimension of the rotation, $D$. 


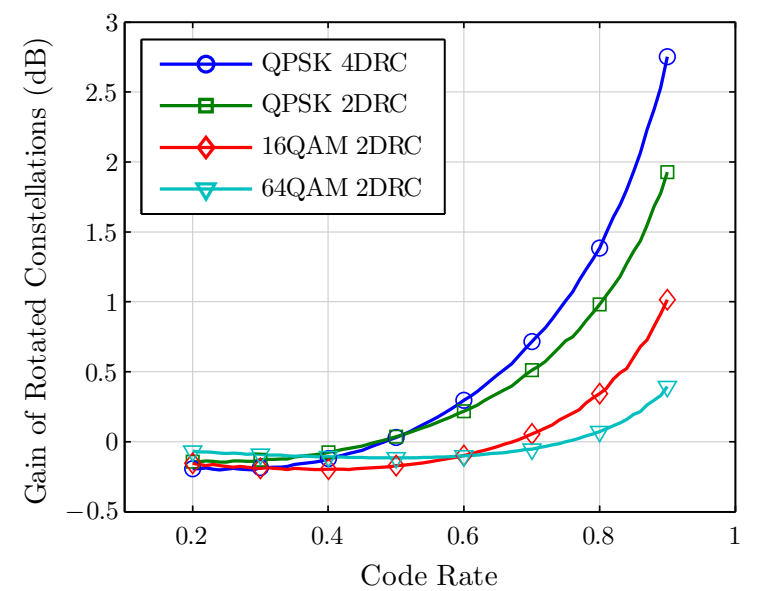

Fig. 4. Gain of rotated constellations in the i.i.d. Rayleigh channel without erasures.

\section{INVESTIGATION BASED ON MUTUAL INFORMATION}

\section{A. Methodology}

In order to evaluate the performance of BICM systems with rotated constellations from a generic point of view (e.g. independently of the particular FEC code), we adopt an information-theoretic approach based on mutual information. Under the assumption of full channel state information (CSI) at the receiver side and ideal infinite interleaving (i.e. an interleaver with length much longer than the coherence time of the channel), the D-dimensional received signal $\mathbf{y}$ in a flat fast fading channel is given by:

$$
\mathbf{y}=\mathbf{H} \mathbf{x}+\mathbf{w}
$$

where $\mathbf{x}=\left[x_{1}, x_{2}, \ldots, x_{D}\right]^{\top}$ is the transmitted symbol, $\mathbf{H}=\operatorname{diag}\left(h_{1}, h_{2}, \ldots, h_{D}\right)$ is the channel matrix with independent fading coefficients in its diagonal, and $\mathbf{w}=$ $\left[w_{1}, w_{2}, \ldots, w_{D}\right]^{\top}$ is the noise vector with independent identically distributed (i.i.d.) circularly symmetric complex Gaussian elements of zero mean and variance $\sigma_{w}^{2}$.

In this case, the capacity obtained by using BICM, $C_{\mathrm{BICM}}$, can be computed as the mutual information $I$ between the set of transmitted code bits $c_{l}, l=1, \ldots, m$ and the received signal $\mathbf{y}$, conditioned by the channel matrix $\mathbf{H}$. With i.i.d. uniform code bits, this is given by [2]:

$$
\begin{aligned}
C_{\mathrm{BICM}} & =\sum_{l=1}^{m} I\left(c_{l} ; \mathbf{y} \mid \mathbf{H}\right) \\
& =m-\sum_{l=1}^{m} \mathrm{E}_{\mathbf{x}, \mathbf{y}, \mathbf{H}}\left\{\log _{2} \frac{\sum_{\mathbf{x}^{\prime} \in \Psi} f\left(\mathbf{y} \mid \mathbf{x}^{\prime}, \mathbf{H}\right)}{\sum_{\mathbf{x}^{\prime} \in \chi_{l}^{b}} f\left(\mathbf{y} \mid \mathbf{x}^{\prime}, \mathbf{H}\right)}\right\},
\end{aligned}
$$

where $b \in\{0,1\}$ is equiprobable and $\chi_{l}^{b}$ denotes the set of rotated symbols in the D-dimensional rotated constellation $\Psi$ for which the code bit $c_{l}$ equals $b$. In the case of the soft-output maximum a posterior (MAP) demodulator,

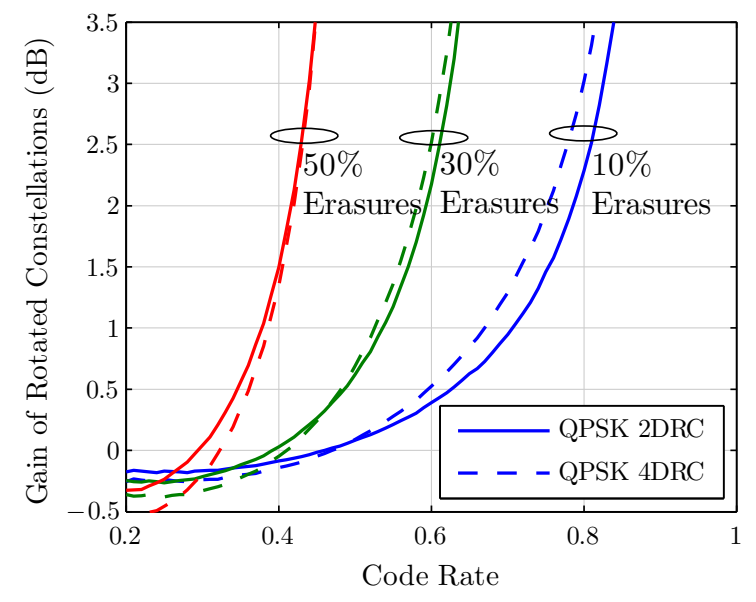

Fig. 5. Gain of rotated constellations in the i.i.d. Rayleigh channel with $10 \%, 30 \%$ and $50 \%$ of erasures.

the conditional probability density function (pdf) $f(\mathbf{y} \mid \mathbf{x}, \mathbf{H})$ corresponds to [3]:

$$
f(\mathbf{y} \mid \mathbf{x}, \mathbf{H})=\frac{1}{\pi \sigma_{w}^{2}} \exp \left(-\frac{\|\mathbf{y}-\mathbf{H} \mathbf{x}\|^{2}}{\sigma_{w}^{2}}\right),
$$

It should be noted that, for any given constellation with spectral efficiency $m$, the BICM capacity determines the FEC code rate $R_{c}$ that is needed in order to achieve error-free communication for any value of carrier to noise ratio (CNR) ${ }^{1}$. In particular, the code rate can be derived from the BICM capacity as $R_{c}=C_{\mathrm{BICM}} / m$. Using the expression in (3), we have computed the code rates for a wide range of CNR values with 4DRC, 2DRC and non-rotated constellations in the i.i.d. Rayleigh channel. This means that each component $h_{i}$ of the channel matrix $\mathbf{H}$ is modelled with uniform phase and Rayleigh amplitude. In addition to Rayleigh fading, we have introduced the presence of erasures as described in [15].

\section{B. Information-Theoretic Results}

In Fig. 4, we represent the gain of 2DRC and 4DRC in terms of CNR compared to non-rotated constellations for code rates ranging between 0.2 and 0.9 in the i.i.d Rayleigh channel without erasures. In the case of $4 \mathrm{DRC}$, we consider QPSK since this is the only configuration supported in the DVBNGH standard. The results show that, broadly speaking, the additional diversity introduced by rotated constellations into the modulation scheme improves the performance of BICM for higher code rates, whereas for lower code rates, it is preferable to rely on the error-correction capabilities of the FEC code. In this manner, rotated constellations do not provide a positive gain if the code rate is low enough, and in fact, they lower the system performance in around 0.1-0.2 dB. The gain of rotated constellations increases with higher code rates depending on the constellation, and is higher than $0 \mathrm{~dB}$ for code rates starting at $0.5(1 / 2), 0.67(2 / 3)$, and 0.75 (3/4) with QPSK,

\footnotetext{
${ }^{1}$ Under the assumption of normalized transmit symbols $\mathrm{E}\left[x x^{\top}\right]=1$, the $\mathrm{CNR}$ is equivalent to the inverse of the noise variance $\mathrm{CNR}=1 / \sigma_{w}^{2}$
} 


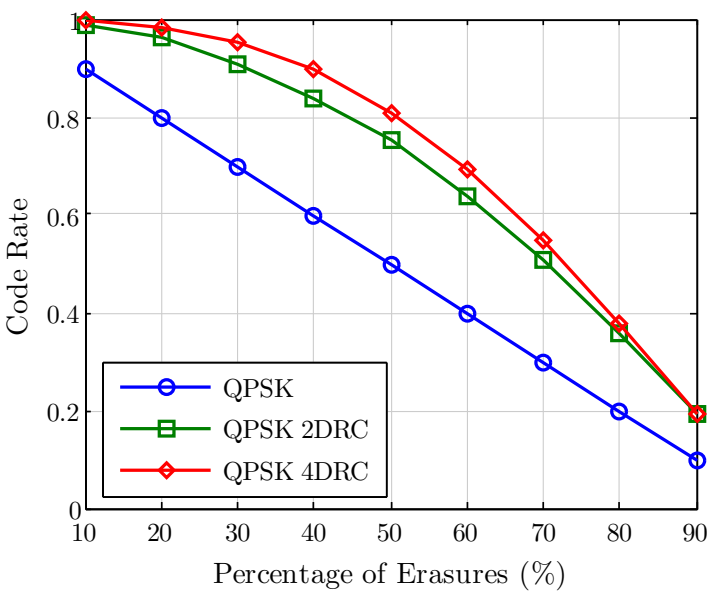

Fig. 6. Maximum code rate supported in the i.i.d. Rayleigh channel according to the percentage of erasures.

16QAM, and 64QAM, respectively. We can also see that the gain is largest with QPSK and decreases with higher order constellations. On the other hand, 4DRC outperform 2DRC for code rates higher than 0.5 with QPSK. The improvement of 4DRC compared to 2DRC increases with the code rate and can be as large as $0.8 \mathrm{~dB}$.

In Fig. 5, we compare the CNR gain of 2DRC and 4DRC with QPSK in the i.i.d Rayleigh channel when a $10 \%, 30 \%$ and $50 \%$ of the information symbols are erased. Despite the fact that erasure ratios lower than $15 \%$ were considered during the standardization of DVB-T2 to model the reception in SFN, a higher percentage of erasures can be realistic in the context of long TI and TFS. According to the results, the gain of rotated constellations increases with the percentage of erasures. Also, the higher the percentage of erasures, the lower the code rate for which rotated constellations achieve a positive gain. It is important to point out that the presence of erasures limits the maximum code rate for which error-free communication is possible. With non-rotated constellations, a 10\%, 30\% and $50 \%$ of erasures require a code rate lower than $0.9,0.7$ and 0.5 respectively in order to repair the loss of information. The additional diversity introduced by rotated constellations diminishes the information loss caused by erasure events, and allows error-free communication with higher code rates. Consequently, the gain of rotated constellations tends to infinity when the code rate approaches the highest value required by non-rotated constellations.

Fig. 6 depicts the highest code rate that is supported in BICM systems to achieve error-free communication in the i.i.d Rayleigh channel depending on the percentage of erasures. Without rotated constellations, we can see that the code rate is given by $1-P_{e}$, where $P_{e}$ is the ratio of erasures. With rotated constellations, this is no longer the case, as they support significantly higher code rates for the same percentage of erasures. The results in Fig. 6 can also be interpreted as the maximum percentage of erasures that are tolerated for any code rate.

\section{Results Based on Physical Layer Simulations}

\section{A. Simulation Parameters}

For the evaluation of 2DRC and 4DRC in DVB-NGH systems, we have employed physical layer simulations. According to the methodology followed in the standardization process of DVB-NGH, the simulations employ perfect channel estimation and soft-output MAP demodulation. Although the performance gains of rotated constellation might be affected by the use of imperfect channel estimation and sub-optimum demodulators, this approach allows a more direct comparison between the simulation and the information theoretical results. This channel is representative of mobile reception for Doppler frequencies above $10 \mathrm{~Hz}$. The results have been obtained for $33 \mathrm{~Hz}$ of Doppler $\left(f_{d}\right)$, which corresponds to a user velocity of $60 \mathrm{~km} / \mathrm{h}$ at a frequency carrier of $600 \mathrm{MHz}$. For the investigation of time diversity with long TI, we have included the presence of shadowing in the TU6 channel. In particular, we have assumed a user moving at constant velocity across a lognormal CNR map, which is defined by its standard deviation $(\sigma)$ and its correlation distance $\left(d_{\text {corr }}\right)$ as described in [16]. The shadowing model outputs CNR values that correspond to the shadowing experimented by the signal every $100 \mathrm{~ms}$. For the simulations, the model has been configured with $\sigma=5.5 \mathrm{~dB}$ and $d_{\text {corr }}=20 \mathrm{~m}$.

In the case of TFS, it is necessary to include the signal variability between different RF channels in the UHF band. The bandwidth of each RF channel is assumed to be $8 \mathrm{MHz}$, which is the typical value used in the UHF band for TV systems in Europe. We assume that there is no correlation of fast fading between RF channels, whereas there is a certain correlation of shadowing depending on the channel separation, $f_{s}$. In addition to fast fading and shadowing, we consider the presence of a power imbalance caused, among other factors, by the frequency dependency of the antenna aperture and the path loss [17]. The correlation of shadowing in the frequency domain and the power imbalance between channels have been parameterized according to the statistical model presented in [18]. This is based on field measurements performed by the Swedish network operator Teracom. It is important to note that the combination of TFS and rotated constellations can be applied with the same effectiveness to other bandwidths commonly used for TV systems, such as $6 \mathrm{MHz}$ or $7 \mathrm{MHz}$. Although the gain of TFS depends marginally on the channel bandwidth, it is mainly determined by the separation between the channels that are combined in the same multiplex.

\section{B. Time Diversity with Long TI}

We first investigate the utilization of rotated constellations to improve the reception robustness (time diversity) with long TI. In the left side of Fig. 7, we can see the frame error ratio (FER) curves the BBFER curves corresponding to intra-frame interleaving (TI $200 \mathrm{~ms}$ ) and inter-frame interleaving (TI $10 \mathrm{~s}$ ) with uniform and uniform-late configurations. In particular, the uniform-late configuration carries a $50 \%$ of information in the late frame and is referred to as UL $50 \%$. The results show that the gain of rotated constellations not only depends on the code rate, but also on the interleaving duration and the type 

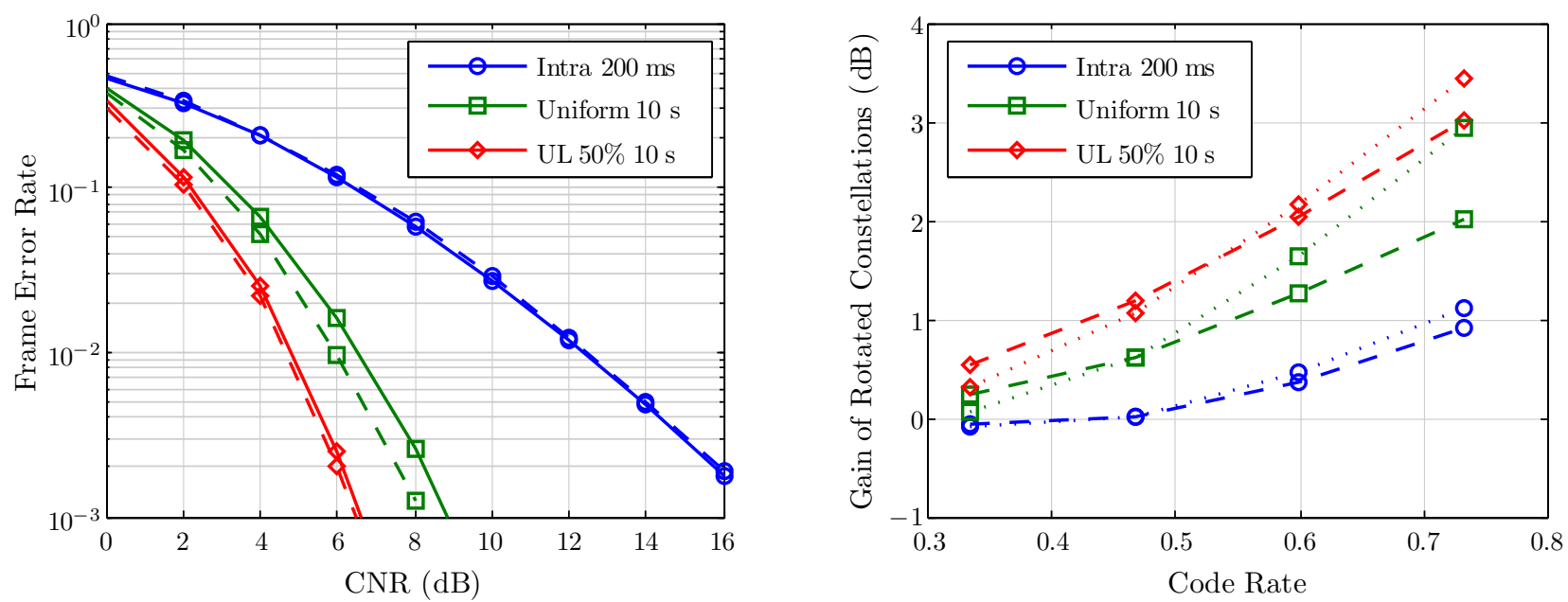

Fig. 7. Performance of DVB-NGH with different configurations of TI in the TU6 channel with shadowing $\left(f_{d}=33 \mathrm{~Hz}, \sigma=5.5 \mathrm{~dB}\right.$ and $\left.d_{\text {corr }}=20 \mathrm{~m}\right)$. On the left, FER curves with QPSK 1/3. On the right, gain of rotated constellations with QPSK. Dashed lines correspond to 2DRC and dotted lines to 4DRC.
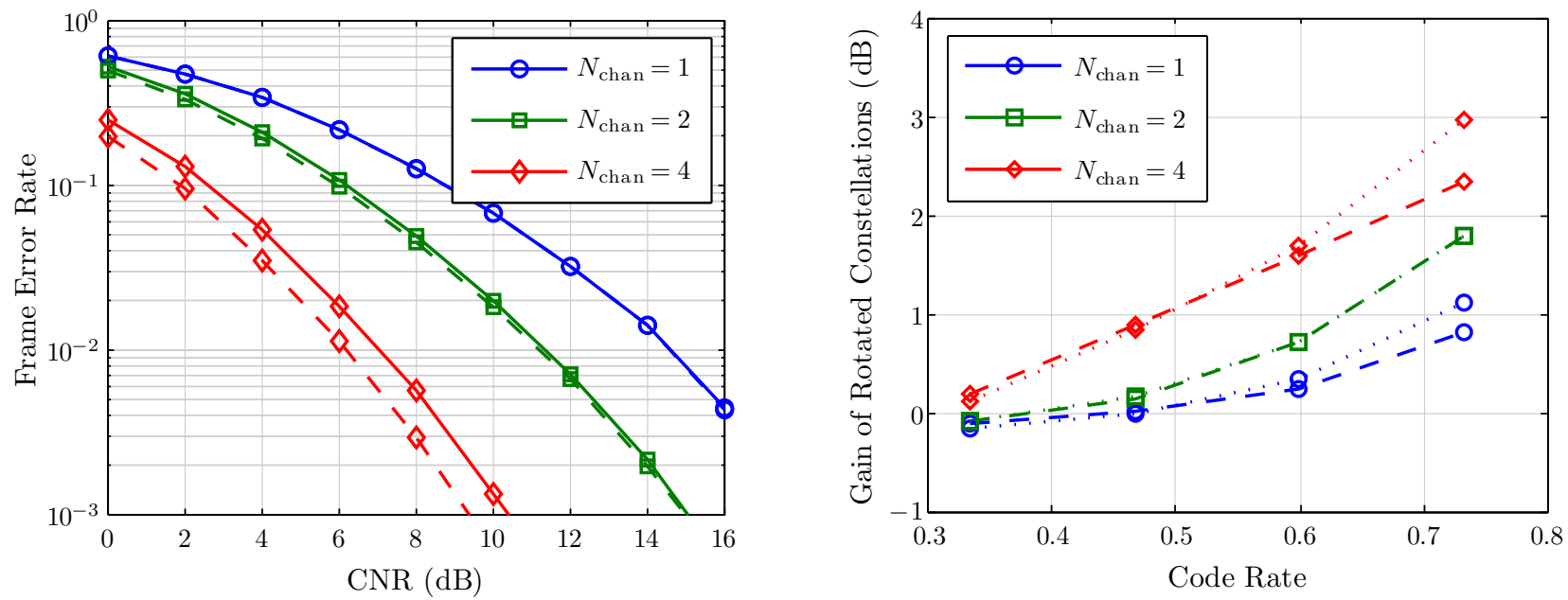

Fig. 8. Performance of DVB-NGH with different configurations of TFS in the TU6 channel with shadowing $\left(f_{d}=33 \mathrm{~Hz}, \sigma=5.5 \mathrm{~dB}, d_{\mathrm{corr}}=20 \mathrm{~m}\right.$ and $f_{s}=100 \mathrm{MHz}$ ). On the left, FER curves with QPSK 7/15. On the right, gain of rotated constellations with QPSK. Dashed lines correspond to 2DRC and dotted lines to 4DRC.

of interleaving. In particular, the gain of rotated constellations is larger with uniform-late configurations, as in this case, they can compensate for the worse time diversity that is achieved with non-uniform interleaving.

The results in the right side of Fig. 7 show the gain of 2DRC and 4DRC for code rates between $1 / 3$ and 11/15. We can see that the gains of rotated constellations with intraframe interleaving are very similar to the information-theoretic values presented in Fig. 6. This is explained by the fact that, with interleaving durations in the order of hundreds of milliseconds, rotated constellations can only counter the presence of fast fading in the received signal. In contrast, the gain of rotated constellations is significantly larger with interframe interleaving, as in this case, the interleaving duration is long enough to compensate for fast fading and shadowing. On the other hand, 4DRC constellations outperform 2DRC with code rates higher than $1 / 2$ in the three configurations of TI. It is interesting to note that the advantage of 4DRC over 2DRC is largest with uniform interleaving, since this achieves a better distribution of components from a time diversity point of view.

\section{Frequency Diversity with TFS}

We now focus on the gain of rotated constellations when the inter-frame interleaving extends over multiple RF channels by means of TFS. In the left side of Fig. 8, we can see the FER curves corresponding to one, two and four RF channels with $f_{s}=100 \mathrm{MHz}$. Therefore, the extent of interleaving in the frequency domain is $8 \mathrm{MHz}, 100 \mathrm{MHz}$ and $300 \mathrm{MHz}$, respectively. Similarly to the case with long TI, the gain of rotated constellations with TFS does not depend only on the code rate, but also on the extent of interleaving in the frequency domain. In this case (QPSK 7/15), the gain varies between $0 \mathrm{~dB}$ with one RF channel up to almost $1 \mathrm{~dB}$ when the interleaving extends across four RF channels. 

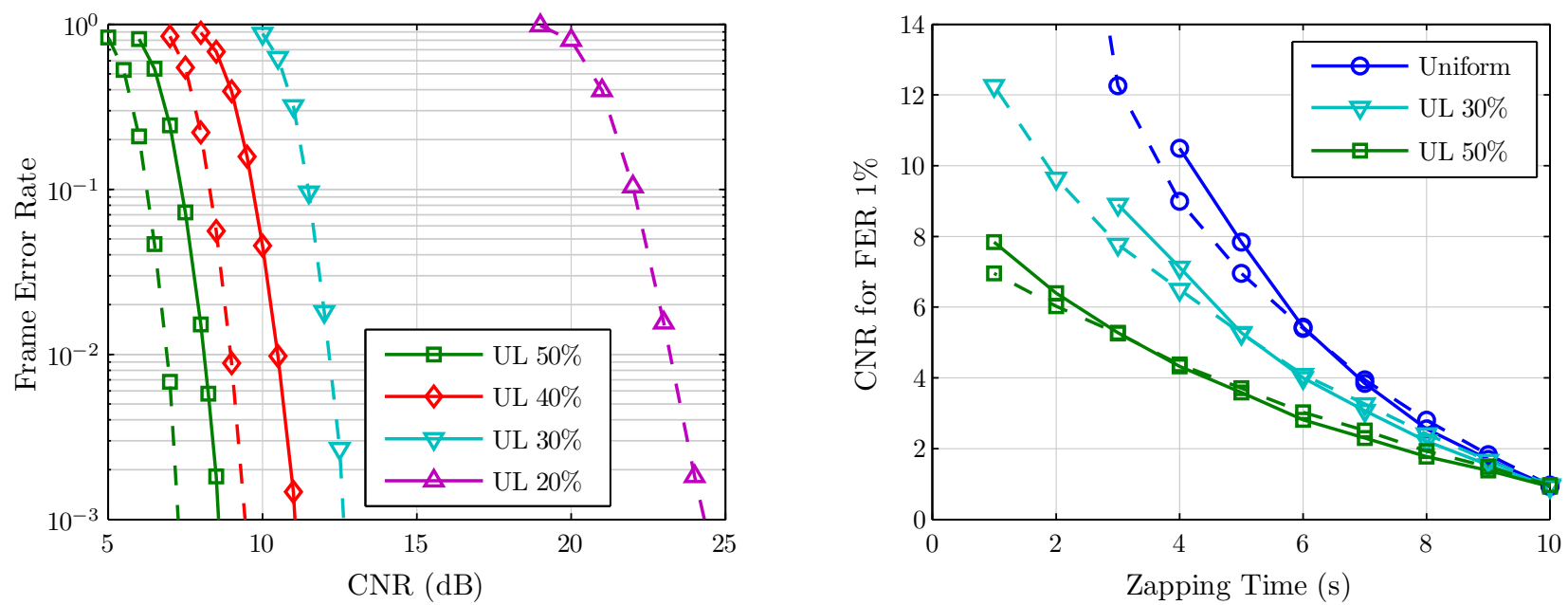

Fig. 9. Zapping performance of DVB-NGH (QPSK 1/3) with different configurations of TI (interleaving duration $10 \mathrm{~s})$ in the TU6 channel $\left(f_{d}=33 \mathrm{~Hz}\right)$. On the left, FER curves with fast zapping after the first information frame. On the right, reception robustness according to the zapping time. Dashed lines correspond to 2DRC.

The results in the right side of Fig. 8 show the gain of 2DRC and 4DRC for code rates between $1 / 3$ and $11 / 15$. As can be seen in the figure, the gain of rotated constellations with TFS grows larger with the code rate and the number of channels. It should be pointed out that, as with long TI, the utilization of rotated constellations with TFS improves the performance of DVB-NGH with code rates as low as $1 / 3$. It is also interesting to note that while 4DRC generally outperform 2DRC, they provide the same performance with two RF channels. This indicates that in order to take advantage of the higher dimensionality of 4DRC with TFS, it is necessary to extend the interleaving across more than two RF channels.

\section{Zapping Performance with Long TI}

In addition to increase the diversity gains of long TI and TFS in the time and frequency domains, rotated constellations can be used to lower the zapping time in DVB-NGH systems. In the left side of Fig. 9, we focus on the reception robustness after receiving the first information frame (zapping time lower than $1 \mathrm{~s}$ ) when the interleaving duration is $10 \mathrm{~s}$. The results show the FER curves corresponding to different uniform-late configurations with QPSK and code rate 1/3. It is important to point out that, in the case of uniform-late interleaving, robust code rates such as $1 / 3$ are generally used to enable low zapping times. The uniform-late configurations selected for the simulations carry a $50 \%, 40 \%, 30 \%$ and $20 \%$ of information in the late frame, and are refered to as UL 50\%, UL $40 \%$, UL $30 \%$ and UL $20 \%$ respectively. Uniform-late configurations with a lower percentage of information in the late frame result in a higher number of erasures at the input of the FEC decoder after the reception of the first frame, and therefore, require a higher CNR to decode the service. The results show that 2DRC (represented in dashed lines) improve the robustness with the UL $50 \%$ and UL $40 \%$ configurations $(50 \%$ and $60 \%$ of erasures) in more than $1 \mathrm{~dB}$. Moreover, they enable the reception with the UL $30 \%$ and UL $20 \%$ configurations
(70\% and $80 \%$ of erasures), which is not possible with nonrotated constellations. This is consistent with the informationtheoretic results presented in Fig. 6, where it is shown that the combination of code rate $1 / 3$ and 2DRC can support a percentage of erasures as high as $80 \%$.

In the right side of Fig. 9, we compare the performance of non-rotated constellations and 2DRC according to the zapping time when the interleaving duration is $10 \mathrm{~s}$. The longer the zapping time, the lower the CNR that is required to decode the service, since a larger percentage of information is available at the receiving side. After $10 \mathrm{~s}$ of zapping time, the receiver has all the information for start decoding the service, and thus, the highest protection is achieved. In the figure, we can see that rotated constellations are very useful to enable shorter zapping times in DVB-NGH, even for code rates as low as $1 / 3$. For example, the utilization of 2DRC lowers in about $2 \mathrm{~s}$ the minimum zapping time that is possible with the uniform and UL $30 \%$ configurations.

\section{CONCLUSIONS}

In this paper we have investigated the 2DRC and 4DRC adopted in the DVB-NGH standard, as well as the gains that can be achieved by combining rotated constellations with long TI an TFS. To this purpose we have used an informationtheoretic approach based on the mutual information together with physical layer simulations in DVB-NGH systems. The information-theoretic results show that rotated constellations improve the performance of BICM when the code rate is high (e.g. > 1/2 with QPSK), whereas for low code rates, nonrotated constellations provide better results.

While the physical layer simulations corroborate the information-theoretic results for interleaving durations in the order of hundreds of milliseconds, they show that the gain of rotated constellations is significantly higher with interleaving durations up to several seconds. The reason is that, with long TI, rotated constellations can compensate for the presence 
of fast fading and shadowing in the received signal. The performance of rotated constellations depends not only on the interleaving duration, but also on the type of interleaving (uniform or uniform-late). In particular, the gain of rotated constellations increases with uniform-late configurations that sacrifice overall robustness (time diversity) to reduce the zapping time. Similarly to the case of long TI, rotated constellations provide larger gains with TFS, as in this case, they can compensate for the large signal variations that exist between different RF channels in the UHF band.

The information-theoretic results and the physical layer simulations reveal that rotated constellations increase the resilience of BICM systems against erasures. On one hand, this improves the reception robustness of long TI and TFS against the presence of signal outages in the time and frequency domain. On the other hand, it can reduce the zapping time with long TI in several seconds.

The results presented in this paper also show that 4DRC outperform 2DRC with code rates higher than $1 / 2$ in the case of QPSK, and that the advantage of 4DRC over 2DRC depends on the interleaving duration, the type of interleaving, and also on the number of RF channels used in TFS. More precisely, the improvement of 4DRC compared to $2 \mathrm{DRC}$ is larger with interleaving durations in the order of seconds, uniform interleaving, and when the number of RF channels is four or more.

\section{REFERENCES}

[1] F. Selgert, "Commercial requirements for DVB-NGH," DVB Document CM-NGH015R1, 2009.

[2] G. Caire, G. Taricco, and E. Biglieri, "Bit-interleaved coded modulation," IEEE Transactions on Information Theory, vol. 44, no. 3, pp. 927-946, 1998.

[3] J. Boutros and E. Viterbo, "Signal space diversity: A power and bandwidth efficient diversity technique for the rayleigh fading channel," IEEE Transactions on Information Theory, vol. 44, no. 4, pp. 14531467, 1998.

[4] S. P. Herath, "Rotated multi-D constellations in Rayleigh fading: Mutual information improvement and a pragmatic approach for near-capacity performance in high-rate regions," in IEEE Wireless Communications and Networking Conference (WCNC'11), Cancun, Mexico, Mar. 2011.

[5] Frame structure channel coding and modulation for a second generation digital terrestrial television broadcasting system (DVB-T2), ETSI Std. EN 302 755, Rev. 1.2.1, 2011.

[6] M. Eroz, F. W. Sun, and L. N. Lee, "An innovative low-density paritycheck code design with near-shannon-limit performance and simple implementation," IEEE Transactions on Communications, vol. 54, no. 1, pp. 13-17, 2006.

[7] Implementation guidelines for a second generation digital terrestrial television broadcasting system (DVB-T2), ETSI Std. TR 102 831, Rev. $0.10 .4,2010$.

[8] C. A. Nour and C. Douillard, "Rotated QAM constellations to improve BICM performance for DVB-T2," in IEEE International Symposium on Spread Spectrum Techniques and Applications (ISSSTA'08), Bologna, Italy, Aug. 2008.

[9] Next Generation broadcasting system to Handheld, physical layer specification (DVB-NGH), DVB BlueBook A160, DVB Std., 2012.

[10] D. Gómez-Barquero, Next Generation Mobile Broadcasting. CRC Press, 2013.

[11] D. Gómez-Barquero, D. Gozálvez, and N. Cardona, "Application layer FEC for mobile TV delivery in IP datacast over DVB-H systems," IEEE Transactions on Broadcasting, vol. 55, no. 2, pp. 396-406, 2009.

[12] M. Makni, J. Robert, and E. Stare, "Performance analysis of time frequency slicing," in ITG Conference on Electronic Media Technology (CEMT'11), Dortmund, Germany, Mar. 2011.

[13] Q. Xie, J. Song, K. Peng, F. Yang, and Z. Wang, "Coded modulation with signal space diversity," IEEE Transactions on Wireless Communications, vol. 10 , no. 2 , pp. 660-669, 2011.
[14] L. Vangelista et al., "Key technologies for next-generation terrestrial digital television standard DVB-T2," IEEE Communications Magazine, vol. 47 , no. 10 , pp. 146-153, 2009.

[15] C. A. Nour and C. Douillard, "Improving BICM performance of QAM constellations for broadcasting applications," in 5th International Symposium on Turbo Codes and Related Topics, Lausanne, Switzerland, Sep. 2008.

[16] S. Saunders and A. Aragón-Zabala, Antennas and Propagation for Wireless Communication Systems, 2nd ed. Wiley, 2007.

[17] "Frequency and network planning aspects of DVB-T2," Technical Report, EBU Tech 3348, 2012.

[18] J. J. Giménez, D. Gozálvez, D. Gómez-Barquero, and N. Cardona, “A statistical model of the signal strength imbalance between RF channels in a DTT network," Electronics Letters, vol. 48, no. 12, pp. 731-732, 2012. 